Meta

Journal des traducteurs

Translators' Journal

\title{
Le fonctionnement spécifique de la mémoire de travail en traduction
}

\section{Alexandra Kosma}

Volume 52, numéro 1, mars 2007

Traductologie : une science cognitive

URI : https://id.erudit.org/iderudit/014716ar

DOI : https://doi.org/10.7202/014716ar

Aller au sommaire du numéro

Éditeur(s)

Les Presses de l'Université de Montréal

ISSN

0026-0452 (imprimé)

1492-1421 (numérique)

Découvrir la revue

Citer cet article

Kosma, A. (2007). Le fonctionnement spécifique de la mémoire de travail en traduction. Meta, 52(1), 22-28. https://doi.org/10.7202/014716ar

\section{Résumé de l'article}

Nous avons réalisé une étude sur le fonctionnement cognitif des traducteurs et plus précisément sur le fonctionnement spécifique de la mémoire de travail lors de l'opération traduisante. Nous avons utilisé comme référence de base le modèle de la mémoire de travail proposé par Baddeley (2000), qui est un des modèles les plus pertinents à l'heure actuelle. Le protocole expérimental proposé aux six sujets traducteurs mettait en comparaison les trois sous-composantes du modèle de Baddeley et a pu montrer une petite différenciation du buffer épisodique par rapport aux composantes de la boucle phonologique et du calepin visuo-spatial. À savoir, lors de la traduction, les sujets ont du mal à retenir des informations « épisodiques », et ceci perturbe la réalisation de cette double tâche. Éventuellement, cette difficulté révélerait l'existence des processus complexes mis en place par le buffer épisodique, qui nécessiteraient beaucoup de ressources attentionnelles, et qui joueraient un rôle essentiel dans le bon déroulement de la traduction. Nous pensons ainsi que cette composante est celle qui s'avère la plus précieuse pour la mise en oeuvre de l'activité de la traduction.
Ce document est protégé par la loi sur le droit d'auteur. L'utilisation des services d’Érudit (y compris la reproduction) est assujettie à sa politique d'utilisation que vous pouvez consulter en ligne.

https://apropos.erudit.org/fr/usagers/politique-dutilisation/ 


\title{
Le fonctionnement spécifique de la mémoire de travail en traduction
}

\author{
ALEXANDRA KOSMA \\ Université ionienne, Corfou, Grèce \\ Université de Caen, Caen, France
}

\begin{abstract}
RÉSUMÉ
Nous avons réalisé une étude sur le fonctionnement cognitif des traducteurs et plus précisément sur le fonctionnement spécifique de la mémoire de travail lors de l'opération traduisante. Nous avons utilisé comme référence de base le modèle de la mémoire de travail proposé par Baddeley (2000), qui est un des modèles les plus pertinents à l'heure actuelle. Le protocole expérimental proposé aux six sujets traducteurs mettait en comparaison les trois sous-composantes du modèle de Baddeley et a pu montrer une petite différenciation du buffer épisodique par rapport aux composantes de la boucle phonologique et du calepin visuo-spatial. À savoir, lors de la traduction, les sujets ont du mal à retenir des informations «épisodiques», et ceci perturbe la réalisation de cette double tâche. Éventuellement, cette difficulté révélerait l'existence des processus complexes mis en place par le buffer épisodique, qui nécessiteraient beaucoup de ressources attentionnelles, et qui joueraient un rôle essentiel dans le bon déroulement de la traduction. Nous pensons ainsi que cette composante est celle qui s'avère la plus précieuse pour la mise en œuvre de l'activité de la traduction.
\end{abstract}

\section{ABSTRACT}

Our study was based on the cognitive functions of translators and, in particular, on the specific function of working memory in translation. We used as a reference Baddeley's model of working memory (2000), which is currently one of the most influential models on the subject. A comparison between the three components of Baddeley's model of working memory reveals that the episodic buffer is slightly diversified from the phonological loop and the visuospatial sketchpad. That is, the translators find it difficult to keep in memory "episodic" information and therefore they cannot bring to an end the double task. This difficulty could possibly be an indication of the episodic's buffer complex processes, which demand great attentional resources and play a key role during the task of translation. Thus, we find that this component is probably the most essential for the act of translating.

\section{MOTS-CLÉS/KEYWORDS}

mémoire de travail, modèle de Baddeley, buffer épisodique, fonctionnement cognitif

L'acte de traduire est une opération assez complexe et pourtant peu étudiée d'un point de vue expérimental. Nos connaissances actuelles sur ses différentes étapes, de même que sur les fonctions cognitives et mnésiques qui sont mises en place chez les traducteurs sont extrêmement limitées et se basent surtout sur un contexte théorique. Dans le cadre du master conjoint franco-hellénique «Sciences de la Traduction-Traductologie et Sciences cognitives", nous avons réalisé une étude, qui visait à mettre en évidence des aspects mal connus ou, jusqu'à présent, inaperçus de l'acte traductionnel, en s'appuyant sur une approche expérimentale. L'intérêt de notre travail a été centré sur le fonctionnement cognitif des traducteurs et, en particulier, sur le fonctionnement d'un certain type de mémoire à court terme, appelé mémoire de travail (MDT). 
Le terme "mémoire de travail» a été apparemment inventé par Miller, Galanter et Pribram (1960). Il a été ensuite adopté par Baddeley et Hitch (1974), dans le but de différencier leur modèle mnésique à composantes multiples, des modèles de mémoire à court terme existants, qui parlaient d'une mémoire unitaire. Au cours du temps, cette notion a eu quelques modifications et ajouts supplémentaires qui mettaient en évidence un paramètre essentiel: la manipulation des informations stockées. Ainsi, en 1992 Baddeley définit la mémoire de travail comme «un système de capacité limitée, qui permet le stockage temporaire et la manipulation des informations qui sont nécessaires pour la réalisation des tâches cognitives complexes, telles que la compréhension, l'apprentissage et le raisonnement.

Selon le modèle de Baddeley et Hitch, proposé en 1974, le système de la mémoire de travail consisterait en trois composantes différentes: la composante principale, appelée administrateur central, serait un système de contrôle attentionnel, qui superviserait et coordonnerait les fonctions de deux sous-systèmes auxiliaires, soit celles de la boucle phonologique, considérée comme responsable du stockage et du traitement des informations provenant du langage (informations verbales et auditives) et du calepin visuo-spatial, considéré comme responsable de l'établissement, du stockage et de la manipulation des images mentales (informations visuelles, spatiales et probablement kinesthésiques).

Pendant une trentaine d'années, le modèle proposé par Baddeley et Hitch a pu offrir une explication pertinente pour une multitude de phénomènes qui s'associent à la mémoire de travail et à ses déficiences. Or, il y avait quelques aspects qui n'étaient pas prévus par le modèle initial et mettaient en question sa cohérence. Par conséquent, il y a six ans, Baddeley a proposé des changements sur son premier modèle, en indiquant des modifications, non seulement aux composantes existantes, mais aussi en modifiant la structure propre du modèle, avec l'ajout d'une composante supplémentaire: le buffer épisodique.

Le buffer épisodique est conçu comme un lieu de stockage à capacité limitée qui est capable de recevoir et regrouper des informations provenant de sources diverses (phénomène de «binding»). En plus, il permet l'interaction de la mémoire de travail avec la mémoire à long terme et fournit ou reçoit par elle des données diverses (Baddeley 2000).

Ce système à trois sous-composantes, coordonné par l'administrateur central, serait impliqué, d'après Baddeley, dans la réalisation des tâches cognitives complexes. La traduction, une tâche cognitive extrêmement complexe, dont les processus ne sont toujours pas clairs, a été l'objet central de notre recherche. En nous basant sur le modèle théorique de Baddeley (2000), un des modèles de la mémoire de travail les plus pertinents à l'heure actuelle, nous avons essayé de délimiter l'implication de ce système mnésique sur l'opération traduisante. Toutefois, il est important de voir comment cette opération est conçue par différents théoriciens de la traduction. Une recherche dans la littérature révèle qu'au sein de la traductologie règne un grand débat. Les points de vue sur les approches traductionnelles appliquées, mais aussi sur la conception de la traduction en tant qu'activité ne convergent pas.

Les principaux courants opposés seraient le courant de la théorie dite linguistique et celui de la théorie dite interprétative. Dans la littérature, la dénomination des différents courants peut varier d'un traductologue à l'autre. Ainsi, chez Newmark (1982) nous retrouvons cette distinction comme l'opposition entre traduction sémantique 
(orientée vers la syntaxe et le contenu sémantique du texte de départ) et traduction communicative (orientée vers la compréhension et la réaction du récepteur), alors que chez Ladmiral (1990) ces deux approches opposées sont décrites comme des attitudes de sourcier et de cibliste. Toutefois, le noyau dur du débat tourne toujours autour du même sujet: la fidélité au sens. D’après la théorie linguistique, la traduction est l'interprétation des signes linguistiques au moyen d'une autre langue (Jacobson, 1963), alors que la théorie interprétative préconise la primauté du sens sur la forme et considère que traduire est un acte de communication et non de linguistique (Seleskovitch 1984). Donc, pour les supporteurs du courant linguistique la traduction serait une «affaire des langues», alors que pour les traductologues en faveur de la théorie interprétative, elle serait un «acte de communication».

La traduction est en effet une activité mentale très complexe qui comporte et met en évidence plusieurs fonctions cognitives. La lecture, la compréhension, et finalement la rédaction ne sont que les étapes les plus importantes, mais ces trois phases ne peuvent pour autant décrire d'une manière exhaustive les opérations mentales qui sont mises en place lors de l'activité de traduire. Une multitude de facteurs extralinguistiques jouent un rôle indispensable pour le bon déroulement de l'opération traduisante et sollicitent entre autres la mise en place de la mémoire de travail. Une décomposition de cette activité cognitive à ses étapes principales pourrait mettre en évidence cette implication. En premier lieu, l'opération traduisante consiste à lire le texte de départ, ensuite, voire en même temps, à le comprendre et finalement à le réexprimer dans la langue d'arrivée.

La lecture implique le traitement des informations visuelles et verbales (par conséquent, implication du calepin visuo-spatial et de la boucle phonologique), la compréhension nécessite l'interaction entre MDT et mémoire à long terme et la formation des images mentales (donc, implication du buffer épisodique et du calepin visuospatial) et la réexpression soit par écrit, soit oralement, pour la traduction à vue, implique le traitement des informations visuelles et verbales ou juste verbales (implication du calepin visuo-spatial et /ou de la boucle phonologique). Nous observons, alors, que la mémoire de travail et ses diverses composantes sont fortement liées à l'acte traductionnel.

Pourtant, quel est le degré d'implication de chaque composante mnésique et laquelle est la plus importante pour l'opération de la traduction? Cette question a été la problématique principale de notre travail. Cependant, nous n'avons pas pu émettre d'hypothèses, puisque dans la littérature actuelle, il n'y a aucune référence sur ce sujet et aucune étude avec une problématique similaire. Par conséquent, afin de répondre à notre question, nous avons dû mettre au point un protocole expérimental, en explorant ce champ thématique à partir de zéro.

Pour cette étude nous avons sélectionné six (6) sujets féminins, dont l'âge moyen était $28,7 \pm 4,01$. Les critères de sélection consistaient en avoir des sujets, dont la langue maternelle est le grec et qui maîtrisent très bien le français, en ayant une formation spécialisée en traduction. Quant à la réalisation des expériences, nous les avons divisées en deux étapes. En premier lieu, nous avons développé un dispositif pour tester l'influence de différentes composantes de la mémoire de travail sur la traduction écrite. En deuxième lieu, nous avons examiné le même phénomène, mais sur la traduction à vue. Le dispositif proposé consistait en quatre tâches expérimentales. La première servait de tâche de contrôle et n'impliquait que la traduction d'un 
texte simple. Pour les trois autres, dans le cadre d'une double tâche, nous avons imposé, au-delà de la traduction d'un texte, une charge mnésique supplémentaire, pour combler les trois sous-composantes mnésiques.

Notre dispositif a été basé sur l'idée qu'une charge mnésique supplémentaire entraînerait une chute dans les performances de la traduction. Ainsi, une composante qui ne s'implique pas beaucoup lors de la traduction ne provoquerait pas de grandes modifications dans les performances des traducteurs, une fois saturée, alors qu'une autre jouant un rôle prépondérant entraînerait une chute de ces performances. Avant le début des expériences, nous avions utilisé la WAIS III de Wechsler pour mesurer l'empan mnésique des sujets. De cette façon, nous avions l'empan exact pour chaque sujet et nous pouvions être sûrs que la double tâche proposée ultérieurement au sujet ne serait ni très facile, ni très dure pour entraîner un débordement de la mémoire à cause d'une surcharge mnésique.

Donc, durant la tâche de contrôle, un texte rédigé en français a été présenté au sujet, et la seule consigne pour le sujet était de faire une traduction du texte dans sa langue maternelle (le grec). Dans cette condition aucune sollicitation supplémentaire de la MDT n'a pas était effectuée.

La deuxième tâche expérimentale proposait de saturer la composante de la boucle phonologique. Le sujet devait traduire un texte français différent de celui utilisé en tâche de contrôle, et, en même temps, il devait garder en mémoire une série de chiffres (correspondant à son empan mnésique), présentée oralement par l'expérimentateur juste avant le début de la traduction. Pour être certain que le sujet ferait son mieux et qu'il s'agissait vraiment d'une double tâche, nous prévenions le sujet qu'il devrait restituer cette série de chiffres à la fin de la réalisation de la traduction dans l'ordre correct.

La troisième tâche expérimentale cherchait à saturer le calepin visuo-spatial. Afin d'arriver à la saturation de cette sous-composante, nous avons utilisé une image abstraite. Nous avons demandé au sujet de la garder bien en mémoire, car juste après la fin de la traduction, il devrait la reconnaître parmi plusieurs images similaires. Ensuite, nous avons présenté au sujet un troisième texte français à traduire en grec et, directement après, nous lui avons présenté cette série d'images abstraites qui contenait aussi l'image présentée initialement.

Enfin, la quatrième tâche expérimentale a été conçue pour saturer le buffer épisodique, et nous avons essayé de le bloquer avec la récitation d'un petit texte avant le début de la tâche. Ce texte contenait des informations factuelles, temporelles et spatiales, c'est-à-dire des informations impliquées dans la création mnésique d'un «épisode». Dès que le petit texte était lu au sujet, nous lui présentions le texte à traduire et ensuite le sujet devait répondre à un questionnaire, lequel évaluerait le degré de rétention des informations du premier texte grâce au buffer épisodique. Notre protocole expérimental était basé sur ce dispositif, commun pour les deux phases des expériences. Or, la différence de nature des deux types de traduction (écrite et à vue) suppose quelques différences méthodologiques. Ainsi, pour la tâche de la traduction écrite, nous devions imposer aux sujets un temps limité. Comme nous nous intéressons aux processus cognitifs se mettant en place dans la MDT, qui est une mémoire à court terme, nous avons imposé quatre minutes pour la réalisation des expériences. En ce qui concerne la traduction à vue, les consignes changeaient en raison de la présentation orale des expériences et l'emploi d'un système oculométrique, 
qui nous donnait des données supplémentaires sur le comportement oculaire des traducteurs.

Toutes les données que nous avons obtenues ont été ensuite regroupées, évaluées et analysées. Pour l'évaluation de performance de chaque composante mnésique, nous avons adopté une échelle discontinue de trois catégories (0-1-2) en fonction de la restitution réussie ou non des informations mnésiques à retenir. Quant à l'évaluation des performances des traducteurs, comme pour la mémoire, nous avons employé une échelle discontinue des trois catégories, appliquée sur cinq niveaux textuels différents (morphosyntaxe, sémantico-lexical, stylistique, pragmatique, organicité). Le critère pour qualifier qu'une performance appartenait à la catégorie 0,1 , ou 2, était basé sur l'importance qu'une éventuelle erreur avait sur la fonction communicative du texte (Kussmaul 1995; Politis 2001) qui venait d'être traduit. Finalement, les données fournies par le système oculométrique concernaient cinq paramètres du comportement oculaire (la durée moyenne des fixations, la fréquence des fixations, le temps d'exploration du texte, le nombre de fixations et la durée de la traduction produite oralement par le sujet) et des stratégies d'exploration.

Pour le traitement statistique de toutes les données tirées par l'évaluation de la traduction et du maintien mnésique, nous avons utilisé le test non paramétrique de «Friedman» de Minitab. Les résultats que nous avons obtenus par l'évaluation des performances mnésiques démontrent que, pendant la traduction à vue, les traducteurs n'ont pas pu réaliser la double tâche et bien restituer les informations lors de la tâche impliquant le buffer épisodique et la boucle phonologique. Pendant la traduction écrite, nous retrouvons les mêmes difficultés juste pour la tâche impliquant le buffer épisodique. L'analyse statistique effectuée en mettant en relation les performances en traduction et le maintien des informations a révélé des effets significatifs pour la tâche de la traduction à vue, mais pas pour la traduction écrite.

Les effets significatifs lors de la traduction à vue concernent une différence statistiquement significative au niveau sémantico-lexical, lorsque nous contrôlons l'ensemble des tâches expérimentales. La probabilité est de l'ordre de $\mathrm{p}=0,040$. Nous obtenons par ailleurs une différence à la limite de la significativité de $\mathrm{p}=0,093$, qui concerne le niveau de la stylistique. Ensuite, de nouveau dans les expériences de la traduction à vue, lorsque nous opposons la tâche de contrôle à la tâche qui implique le buffer épisodique, nous constatons une différence significative au niveau stylistique. La probabilité est de l'ordre de $\mathrm{p}=0,046$. Nous obtenons par ailleurs une différence à la limite de la significativité pour les mêmes tâches expérimentales au niveau sémantico-lexical ( $\mathrm{p}=0,083)$. Même si elle n'est pas significative, elle se distingue nettement de toutes les autres tâches et tous les autres niveaux.

En ce qui concerne les résultats des stratégies d'exploration oculaire des sujets, nous avons constaté qu'il n'y avait pas des différences importantes d'une tâche expérimentale à l'autre. Il y avait une très grande variabilité des parcours suivis par les six sujets pour un même texte, mais cette variabilité ne peut être attribuée qu'au facteur individuel. Par ailleurs, une observation qui paraît intéressante est que le parcours oculaire des sujets semble être corrélé à la stratégie de traduction mise en place par chaque sujet. Nous remarquons, par exemple, que les sujets qui font une traduction dite «mot à mot» en ne cherchant que des correspondances de mots et non de sens, ont un parcours oculaire quasi linéaire, alors que les sujets qui optent pour une stratégie plus synthétique et appliquent l'approche proposée par la théorie interprétative 
ont un parcours beaucoup plus exploratoire (Pestel 2003). Enfin, l'analyse statistique des données concernant le comportement oculaire ne révèle pas d'effet statistique.

Une première interprétation de nos résultats met en évidence le statut particulier de la composante du buffer épisodique lors de la traduction, par rapport aux deux autres composantes mnésiques. Nous remarquons une énorme difficulté de la part des traducteurs à restituer les informations épisodiques, tant pour la phase de la traduction à vue, que pour la phase de la traduction écrite. Ces données nous font penser qu'apparemment le fonctionnement du buffer épisodique utiliserait les mêmes ressources que celles s'activant lors de l'opération traduisante. Il y a alors une surcharge importante pendant la double tâche, qui finit par l'échec presque absolu de nos sujets à restituer les informations épisodiques en traduction à vue, ainsi qu'une restitution extrêmement déficitaire en traduction écrite.

En outre, puisque pour toutes les autres composantes, les sujets ont pu finalement maintenir et restituer les informations avec succès, la composante du buffer épisodique qui fait l'objet d'échecs constants se révèle être la composante la plus importante pour la réalisation de la traduction. Le buffer épisodique comporterait ainsi des structures plus complexes ou demanderait des ressources plus importantes que les autres composantes lors de la traduction. Toutefois, il faut être prudent, car ceci n'est qu'une hypothèse faite à partir d'un petit échantillon et nous manquons de données supplémentaires.

En guise de conclusion, nous pourrions dire que, comme jusqu'à présent il n'y avait pas vraiment beaucoup de recherches sur ce sujet, nous avons dû élaborer un protocole expérimental original, dont l'efficacité n'avait pas encore été testée dans d'autres études. Nous y avons repéré quelques défauts, qui ont compliqué le traitement de nos résultats et ont posé des difficultés quant à nos conclusions finales, par exemple le petit nombre de sujets dans notre échantillon, l'ordre de passation des tâches expérimentales et le non-respect des consignes de la part de quelques traducteurs.

Néanmoins, en même temps, notre sujet offre une nouvelle approche de l'opération traduisante et fourni quelques informations, parmi les premières, sur les fonctions cognitives et mnésiques des traducteurs. En même temps, il peut fournir une critique constructive de différents modèles théoriques de l'acte traductionnel. La nouvelle composante de MDT, proposée par Baddeley, le buffer épisodique, prend une place importante dans ce modèle et semble jouer un rôle essentiel dans le fonctionnement mnésique lors de la traduction. Ainsi, il semblerait que l'opération traduisante n'est pas simplement «une affaire de langues». En outre, maintenant, le champ est ouvert pour la continuation des études sur cette thématique et l'obtention des résultats expérimentaux, qui seraient exploitables tant par la communauté scientifique que par les traducteurs eux-mêmes.

\section{RÉFÉRENCES}

BAdDELEY, A.D. (2000): “The episodic buffer : a new component of working memory?", Trends in Cognitive Sciences 4, p. 417-423.

BADdeley, A.D. (1992): "Working memory", Science 255, p. 556-559.

Baddeley, A.D. and G. Нitch (1974): "Working memory", in Bower, G. A. (eds.), Recent Advances in Learning and Motivation vol.VIII, New York, Academic Press.

JACOBSon, R. (1963): Essais de linguistique générale, Paris, Éditions de Minuit.

Kussmaul, P. (1995): Training the Translator, Amsterdam/Philadelphia, John Benjamins.

Ladmiral, J.-R. (1990) : «La traduction proligère? - Sur le statut des textes qu'on traduit», Meta $35-1$. 
28 MetA, LII, 1, 2007

Miller, G. A., Galanter, E. and K. H. Pribram (1960): Plans and the Structure of Behavior, New York, Holt, Rinehart and Winston.

Newmark, P. (1982): Approaches to Translation, Oxford, Pergamon Institute of English. Pestel,

A. (2003): Du regard au texte: influence de la mise en forme matérielle d'un texte procédural sur les stratégies d'exploration oculaire, Mémoire de maitrise de psychologie, Université de Caen.

Politis, M. (2001): L'évaluation des erreurs en traduction, communication orale au $5^{e}$ Congrès national de linguistique grecque, Université Paris-Sorbonne, 13-15 septembre 2001.

Seleskovitch, D. et M. Lederer (1984): Interpréter pour traduire, Paris, Didier Érudition. 